\section{We need a balanced debate on direct access}

\author{
D. Bridges, ${ }^{1}$ A. Gallie, ${ }^{2}$ S. Howe ${ }^{3}$ and E. Tilling ${ }^{4}$
}

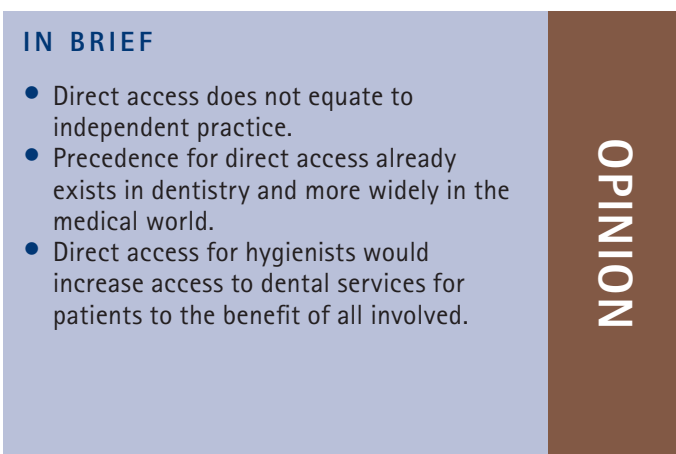

As active members of a group of like-minded dental care professionals campaigning for the establishment of direct access (DA) for dental hygienists (DHs) we read the BDJ editorial Direct line lack of assurance with interest. ${ }^{1}$ However, our interest soon waned and turned to disappointment as it became clear this was to be no balanced debate of the issue.

It is, at best, disappointing to have the anecdotal stories, of what some might perceive as the British Dental Association's (BDA's) protectionist stance, confirmed in print. Even more so when part of this argument seems to be based on apparently erroneous and disingenuous information.

The first point we would take issue with is the assumption that our case for DA is based on the premise that DA equates to independent practice (IP). Dental care professionals (DCPs) have had the right to own and operate their own IPs since April 2006. Some have already done so, even employing dentists. It is apposite to make it absolutely clear that DA is NOT about IP.

The first point we would agree on is that regarding the non-desirability of setting up in IP. We see DA as being very much a part of life in general practice. True, there are some who would like to set up independently but these are few and, as mentioned earlier, we feel most of those that want to have already done so. For many hygienists, DA would merely legitimise the status quo. The main point of DA is to increase

"Registered Dental Hygienist, Gloucester; ${ }^{2}$ Registered Dental Hygienist, London; ${ }^{3}$ Registered Dental Hygienist, Nottingham; ${ }^{4}$ Registered Dental Hygienist, Wedmore

*Correspondence to: David Bridges

Email:dbrdh@me.com

This opinion article was originally submitted as a letter to the Editor and was co-signed by the following Registered Dental Hygienists: C. Chatfield, S. Murray, M. Ross, D. Benton, L. Card, T. Ives, L. Gibbs, K. Govier and J. Stanfield.

Accepted 24 April 2012

DOI: 10.1038/sj.bdj.2012.558

${ }^{\circledR}$ British Dental Journal 2012; 213: 11-12 access to a 'circle of care' - another entry point into professional dental and, indeed, holistic general healthcare.

The second point we would contest is the supposed lack of precedent. The piece reports there is none, save for the anomaly of clinical dental technicians (CDTs) - a group of DCPs who do have DA. This is precedence. It is also deemed that this registrant group have sufficient skills to identify abnormalities and refer onwards to an appropriate healthcare professional. We contend that all the arguments relating to hygienists' apparent lack of training, their apparent lack of diagnosis skills and the possible risk of missed oral cancer all fall at this point. Yet DA antagonists continue to argue that a hygienist, who has been at full time dental school for at least 24 months, treating many patients under supervision, does not have the necessary skills to recognise pathology.

The precedence angle taken in the editorial also seeks to neatly sidestep the precedence that is optometrists, nurse practitioners, midwives, podiatrists and physiotherapists, all of whom have DA to patients without first recourse to a doctor. They all work professionally within their scope and refer as necessary.

The question of competency has been raised many times. It must be remembered that a DH currently spends a minimum of 24 months, including at least 1,200 clinical hours, predominately concentrating on a single subject. It must also be remembered that most students now dual-qualify as hygienists and therapists (DHTs) with a BSc primary degree after three or four years of study. This aside, we accept that DA for newly qualified hygienists is probably not appropriate. Many nuances are gained with experience and therefore, as part of our suggested model, we would propose that a hygienist should have five years of equivalent post qualification experience on the register before receiving entitlement to DA. DHs are registered, indemnified and subject to the same regulatory structure as general dental practitioners (GDPs); while there is some discussion around the ability to diagnose appropriately, it must be borne in mind that the GDPs themselves often do not diagnose many (any) neoplastic lesions in the dental surgery. They refer the patient onwards to those that have suitable expertise and facilities to hand. Current GDC curricula and guidance determine that DHTs must also be able to recognise oral pathology and refer appropriately. This we do daily already.

We understand that BDS undergraduates complete a longer training course. In actuality, however, there are so many disciplines to cover in that time that periodontal diagnosis and training seems to take a low priority. We have heard from BDS undergraduates who make this very point. We, all in our working lives, may have come into contact with young, newly qualified BDS graduates who cannot carry out accurate indices and therefore cannot collect and synthesise the information needed to make an accurate diagnosis. Periodontal therapy and diagnosis take time to perfect and reach a level of 
comfortable familiarity, and we, as hygienists, carry out these tasks all day everyday - we get a lot of practice.

It is simplistic and wrong to suggest that an experienced DH cannot diagnose periodontal disease or recognise abnormalities. Many a DH in general dental practice has to carry out initial periodontal assessments including editing Basic Periodontal Examinations (BPE) passed to them (if they get them) appropriately in line with the current British Periodontal Society's (BSP's) guidelines. They are also deciding on the appropriate treatment plan for their patients. Indeed, one only has to look at various online forums to see the day-today difficulties that DHs face in practice in this respect. Perhaps the GDC should carry out some simple research to assess the extent of this problem; a few simple questions would show that in general practice very few DHs receive any kind of definitive descriptive prescription and usually work in the absence of a diagnosis. We take the recognition of the BSP to allow DHs full membership to be a true and honest recognition of the work done by DHs to recognise, diagnose and successfully treat periodontal disease within scope.

It is a truism that $50 \%$ of the population do not attend a dentist. There are many reasons for this. However, there does seem to be a demand for the periodontal services of hygienists, a demand that has been the basis of a successful business model, namely that of SmilePod (www.smilepod.co.uk). This business initially offered predominantly hygiene services ostensibly by hygienists. Their clinicians are, in fact, mainly dentists and they have now made this clearer.

We have many anecdotal accounts of patients who wish to see a hygienist but not a dentist at a particular time. We know that patients frequently ring practices asking to see a hygienist. They may not have access to one at the practice they attend. Why should a patient have to pay for another examination? It makes no sense and is unfair. Getting a referral letter can be difficult with some GDPs seemingly reluctant to put pen to paper and seeing such an act as tantamount to signing away money. We know of persistent patients who have fought to get a referral. This would seem to run counter to the argument that DA hygienists would confuse the public.

We see DA as a means of drawing more patients into professional preventive care at a time that prevention has never been more important with the increasing awareness of oral/systemic interactions. DA would allow us to work more effectively within a practice setting as a standalone registered health professional that can assess and treat within their own competency referring when and where appropriate.

DA would also make business models including partnership a more realistic proposition for DHs. To apply one of business consultant Chris Barrow's lines: 'It's not about dividing the cake into smaller and smaller pieces. It's a whole new cake!' DA is all about increasing access to professional healthcare in a safe, regulated environment.

What practice principal would turn down the prospect of a new source of patients? Particularly during these tough economic times.

1. Hancocks S. Direct line lack of assurance. Br Dent $J$ 2012; 212: 53

\section{Corrigendum}

\section{General article (BDJ 2012; 212: 243-245)}

'Europe's oldest jaw: Evidence of oral pathology'

In the above general article the following statements should have been included:

1) Following the sentence 'Given these considerations, this specimen is considered highly protected material by the National Research Centre of Human Evolution (CENIEH) and thus strong measures have been imposed to restrict access to it.' (Introduction, page 243), the subsequent clarification should be added:

'Thus, the analysis in this paper was carried out using photographs of the fossil and did at no time involve examination of the fossil itself.

Whilst this paper was in preparation, a detailed paleopathological study of the specimen was carried out and reported by Martinón-Torres et al. ${ }^{32}$,

32. Martinón-Torres M, Martín-Francés L, Gracia A et al. Early Pleistocene human mandible from Sima del Elefante (TE) cave site in Sierra de Atapuerca (Spain): a palaeopathological study. J Hum Evol. 2011; 61: 1-11.

2) The caption for Figure 1 should include the following statement: 'Figure reproduced with the permission of Ricardo Ordoñez, photographer. Spain.' The captions for Figures 2-3 should include the following statement: 'Figure reproduced with the permission of Agencia DiCYT. Spain.'

The authors apologise for any confusion caused. 